\title{
Effect of Radix Ranunculi Ternati Polysaccharide on Immunological Function in Normal Mice
}

\author{
M.S. Miao, W.Y. Xin, M. Bai, L. Guo, S. Tian \& H.L. Liu \\ Henan University of Traditional Chinese Medicine, Zhengzhou, Henan, China, 450008 \\ M.S.Miao \\ Professor, Zhengzhou, Henan, China
}

\begin{abstract}
Objective: To explore the effect of Radix Ranunculi Ternate Polysaccharide (RRTP) on immunological function in normal mice. Methods: The effects of high, medium and low dosage of RRTP were investigated in normal mice by detecting the macrophage percentage, phagocytosis index, formation of hemolysis and hemolytic plaque and lymphocyte transformation. Results: Compared with the physiological saline group, the low dosage RRTP and mushroom polysaccharide (MP) obviously increased the macrophage percentage and the increase was more dramatic in the high and medium dosage RRTP groups. The phagocytic index of abdominal cavity in normal mice was peritoneal macrophages was significantly enhanced by the high and medium dose of RRTP and MP and the enhancement was even more dramatic in the RRTP medium dose group. The formation of hemolysin in normal mice was obviously promoted in the RRTP medium dose and MP groups. The hemolytic plaque formation was also significantly promoted in the MP group and it was even more dramatic in the RRTP high dose and medium dose groups. Conclusion: RRTP enhances immune function of normal mice.
\end{abstract}

KEYWORD: Radix Ranunculi Ternati Polysaccharide; Normal mice; Immunological function

\section{INSTRUCTIONS}

Radix Ranunculi Ternate (RRT) is a common folk herbal medicine, mainly distributed in Xinyang of Henan Province, China. Its taste is bitter sweet, having effects on the livers, lungs and body temperature. Its main functions are to clear toxicity, reduce phlegm and relieve pain and inflammation. RRT has a better curative effect when it has been clinically used to treat scrofulous collapse, tuberculous lymphadenopathy and pharyngitis[1]. Modern research shows that RRT has good effects against bacteria, inflammation and cancer. However, the active ingredients of RRT are still unclear. It's been reported that carbohydrate and oil account for $16 \%$ and $1.2 \%$ of RRT ingredients, respectively, and RRT also contains various alkaloids[2]. Clinically, it is used for the treatment of multiple diseases including lymph node tuberculosis, tuberculosis, strep throat, malaria and cancer embolism. RRT has become one of the three major types of traditional medicine and has caught the attention of researchers in China and other countries[3]. To investigate the characteristics of RRT, we observed the effect of RRTP on immune function in normal mice.

\section{MATERIALS}

\subsection{Instruments and reagents drugs}

AUV - 2000 Spectrophotometer was purchased from Unico (Shanghai) Instrument Co, Ltd. RRT plant belongs to the Mao Lang family, and is usually extracted from the dried roots of Mao Lang Ranunculus ternatus thumb. In this study, RRT was obtained from Kaikaiyuansheng Pharmaceutical Co, Ltd. RRTP was provided by the Institute of chemical laboratory. RRT was crushed and degreased. The obtained powder was then extracted with water, concentrated and precipitated with alcohol, followed by the protein dialysis and vacuum drying process. Mushroom polysaccharide tablets were purchased from Zhejiang Aphelia Pharmaceutical Co, Ltd (batch number 060701). PHA was gain from Sigma.

\subsection{Animals}

Kunming (KM) male and female mice in $18 \sim 22 \mathrm{~g}$ weight (animal certificate No.703003) was provided by the Medical Experimental Animal Center of Hebei province (license number SCXK(Hebei)20031-003). 


\subsection{Statistical analysis}

The SPSS 13.0 statistical software for Windows was used for data analysis. The measurement results were expressed as"mean \pm standard deviation $(\overline{\mathrm{x}} \pm \mathrm{s})$ ". Comparison between groups was performed by the LSD method.

\section{METHODS}

\subsection{Phagocytic percentage and phagocytic index of peritoneal macrophages in mice}

25 female mice and 25 male mice (weight, $18 \sim 22 \mathrm{~g}$ ) were randomly divided into five groups. Four groups were respectively fed with high, medium and low dosage of RRTP solutions and mushroom polysaccharide suspension, and therefore the groups were named as RRTP-HD, RRTP-MD, RRTP-LD and MP, respectively. The animals were administered once daily for seven continuous days. The other group was used as the control, fed with the same volume of saline (Table 1). $1 \mathrm{~h}$ after the last administration, each mouse was injected intraperitoneally with $5 \%$ suspension of chicken erythrocyte saline $(0.4 \mathrm{ml} / \mathrm{each}$ mouse $)$. The mice were then sacrificed after $4 \mathrm{~h}[4] .2 .5 \mathrm{ml}$ of Han's solution was injected intraperitoneally. Mouse abdomen was gently cut into a hole in the peritoneum, and $2 \mathrm{ml}$ of peritoneal fluid was collected, placed in a test tube and mixed. Abdominal droplets were added onto a glass slide (about $1.5 \mathrm{~cm} \times 2 \mathrm{~cm}$ ). Placed the slides on porcelain plates with a wet gauze in and incubated at $37^{\circ} \mathrm{C}$ for $30 \mathrm{~min}$. Regular saline was used to rinse off floating cells. Wright's staining was performed to stain the slides followed by washing with water and drying. Phagocytosis of mouse peritoneal macrophages was observed using a microscope, and the phagocytic percentage and phagocytic index were calculated.

\subsection{Hemolysin and hemolytic plaque-forming assay}

Animal grouping and drug administration were done as described above. On the first day of administration, each mouse was injected intraperitoneally with $5 \%$ suspension of chicken erythrocytes saline $(0.2 \mathrm{ml} / \mathrm{each})$. $2 \mathrm{~h}$ after the last administration, blood samples were taken from each mouse[4]. Serum was obtained by centrifuging and then diluted with physiological saline (1:100). $1 \mathrm{ml}$ of diluted serum was mixed with $0.5 \mathrm{ml}$ of $5 \%$ chicken red blood cell suspension and $0.5 \mathrm{ml}$ of $10 \%$ Guinea pig serum (which was pre-saturated with chicken red blood cells for $6 \mathrm{~h}$ ). The mixture was incubated at $37^{\circ} \mathrm{C}$ and ice water was then used to terminate the reaction. The mixture was incubated at $37^{\circ} \mathrm{C}$ for $30 \mathrm{~min}$ and ice water was then used to terminate the reaction. The serum without additional supplements was used as the blank control. The supernatant after centrifugation was detected in a colorimetric tube at 540nm to determine hemolysin formation. Mice were sacrificed by cervical dislocation after blood extraction. The spleen was collected and homogenated. The spleen cells were re-suspended to a density of $5 \times 106 \mathrm{n} / \mathrm{ml}$. $0.5 \mathrm{ml}$ of spleen cell suspension was mixed with $0.5 \mathrm{ml}$ of $0.2 \%$ chicken red blood cell suspension and $0.5 \mathrm{ml}$ of $10 \%$ guinea pig serum. The spleen suspension without additional supplements was used as the blank control. The mixture was incubated at $37^{\circ} \mathrm{C}$ for $1 \mathrm{~h}$ followed by centrifuging. The obtained supernatant was detected with a colorimeter at $413 \mathrm{~nm}$ to determine hemolytic plaque formation.

\subsection{Mouse lymphocyte transformation test}

Animal grouping and drug administration were performed as mentioned above. Before the Day 3 administration, each mouse received intramuscular PHA $(8 \mathrm{mg} / \mathrm{kg})$ daily. $2 \mathrm{~h}$ after the last administration, tail blood was taken and stained with Swiss dye. Oil immersion observation was used to calculate the lymphocyte transformation rate [5].

\section{RESULTS}

\subsection{Effect of normal mouse peritoneal macrophages on the phagocytic function}

Compared with the normal saline group, the phagocytic percentage of mouse peritoneal macrophages was significantly improved in the RRTP-LD and MP groups $(\mathrm{P}<0.05)$ and the improvement was even more dramatic in the RRTPHD and RRTP-MD groups $(\mathrm{P}<0.01)$. The phagocytic index of peritoneal macrophages was significantly enhanced in the RRTP-HD, RRTP-LD and MP groups $(\mathrm{P}<0.05)$ and the enhancement was even more dramatic in the RRTP-MD group $(\mathrm{P}<0.01)$. These results are listed in Table 1. 
Table 1 Effect of RRTP on the phagocytic function of peritoneal macrophages in mice ( $\bar{x} \pm \mathrm{s})$

\begin{tabular}{|l|l|l|l|l|}
\hline Groups & $\mathrm{n}$ & Dose $(\mathrm{mg} / \mathrm{kg})$ & The phagocytic percentage $(\%)$ & Phagocytic index \\
\hline The physiological saline group & 10 & & $34.3 \pm 4.6$ & $0.38 \pm 0.05$ \\
\hline MP & 10 & 150 & $40.1 \pm 4.2^{*}$ & $0.43 \pm 0.04^{*}$ \\
\hline RRTP-HD & 10 & 450 & $40.2 \pm 3.6^{* *}$ & $0.45 \pm 0.04^{*}$ \\
\hline RRTP-MD & 10 & 300 & $41.2 \pm 5.5^{* *}$ & $0.46 \pm 0.05^{* *}$ \\
\hline RRTP-LD & 10 & 150 & $38.5 \pm 4.1^{*}$ & $0.43 \pm 0.04^{*}$ \\
\hline
\end{tabular}

Note: *compare with the normal saline group $P<0.05, * *$ compare with the normal saline group $P<0.01$

\subsection{Influence on the formation of hemolysin and hemolysis in normal mice}

Compared with the normal saline group, the formation of hemolysin was obviously promoted in the RRTP-MD and MP groups $(P<0.01)$. The hemolytic plaque formation was also significantly promoted in the MP group $(P<0.05)$ and it was even more dramatic in the RRTP-HD and RRTP-MD groups $(P<0.01)$. The results are listed in Table 2 .

Table 2 Effect of RRTP on the formation of hemolysis and hemolytic plaque in normal mice ( $\bar{x} \pm \mathrm{s})$

\begin{tabular}{|l|l|l|l|l|l|}
\hline Groups & $\mathrm{n}$ & Dose $(\mathrm{mg} / \mathrm{kg})$ & $\begin{array}{l}\text { The formation of } \\
\text { hemolysin (OD) }\end{array}$ & The number of cases & $\begin{array}{l}\text { The formation of } \\
\text { hemolytic plaque (OD) }\end{array}$ \\
\hline The physiological saline group & 10 & & $0.304 \pm 0.040$ & 5 & $0.321 \pm 0.044$ \\
\hline MP & 10 & 150 & $0.430 \pm 0.125^{* *}$ & 5 & $0.371 \pm 0.036^{*}$ \\
\hline RRTP-HD & 10 & 450 & $0.330 \pm 0.088$ & 5 & $0.406 \pm 0.081^{* *}$ \\
\hline RRTP-MD & 10 & 300 & $0.387 \pm 0.048^{* *}$ & 5 & $0.405 \pm 0.028^{* *}$ \\
\hline RRTP-LD & 10 & 150 & $0.345 \pm 0.077$ & 5 & $0.352 \pm 0.031$ \\
\hline
\end{tabular}

Note: *compare with the normal saline group $P<0.05$, **compare with the normal saline group $P<0.01$

\subsection{Influence on lymphocyte transformation in normal mice}

Compared with the normal saline group, the large, the lymphocyte transformation was significantly promoted in the MP group $(P<0.05)$ and it was even more dramatic in the RRTP-MD group $(P<0.01)$. The results are listed in Table 3.

Table 3 Effect of RRTP on mouse's lymphocyte transformation $(\bar{x} \pm \mathrm{s}$ )

\begin{tabular}{|l|l|l|l|}
\hline Groups & $\mathrm{n}$ & Dose $(\mathrm{mg} / \mathrm{kg})$ & The lymphocyte transformation rate $(\%)$ \\
\hline The physiological saline group & 10 & & $38.3 \pm 5.2$ \\
\hline MP & 10 & 150 & $43.9 \pm 5.9^{*}$ \\
\hline RRTP-HD & 10 & 450 & $49.8 \pm 8.5^{* *}$ \\
\hline RRTP-MD & 10 & 300 & $53.7 \pm 4.2^{* *}$ \\
\hline RRTP-LD & 10 & 150 & $42.4 \pm 4.9$ \\
\hline
\end{tabular}

Note: *compare with the normal saline group $P<0.05$, **compare with the normal saline group $P<0.01$

\section{ACKNOWLEDGEMENTS}

The research work was supported by Cultivation of innovative talents Colleagues of Henan province (2004-23).

\section{DISCUSSION}

RRT was found in 1950s in Xinyang of Henan Province, China and has been used as a Chinese herbal medicine since then. Although its medical history is short, it has been widely applied in clinic and the effect is significant. To data, studies on RRT have mainly concentrated in its in vitro anti-tumour and anti-tuberculosis functions. RRT prevents and treats tuberculosis and other diseases by regulating the immune function of the body. However, the role of RRT in immune regulation is unclear and under intense study. To evaluate the effects of RRTP on immune function in normal mice, we selected phagocytosis of peritoneal macrophages as the 
nonspecific immune function index, hemolysis and hemolytic plaque as the specific humoral immune index and lymphocyte transformation as the cell immune index, respectively[6].

The experimental results showed that high and middle dose of RRTP significantly increased the phagocytic percentage and phagocytic index of peritoneal macrophages in mice, promoted the formation of serum hemolysin and hemolytic plaque and improved lymphocyte transformation, implicating that RRTP obviously regulates the immune function in normal mice. The polysaccharide content in RRT was high, about $6 \%$. This study also revealed the importance of polysaccharide as the main active constituent of RRT. The findings give quality assessment of herbal medicine objectively and provide an experimental basis for the development and utilization of RRT.

\section{REFERENCES}

[1] The State Pharmacopoeia Commission.

(ed.) 2010 . Ch.P(a). Beijing: Chemical Industry Press.

[2] Hu, Zekai. Liu, Huili. \& Miao, Mingsan. 2010. Radix Ranunculi Ternati Polysaccharide induced mouse model of immune function in immunosuppressed cyclophosphamide. China JMAP.27 (2):89.

[3] Li, Rui. \& Yin, Chunping. 2014Progress in research of traditional Chinese Medicine Radix Ranunculi ternati. China Pharmacist.17(3):489-490.

[4] Zhang, Zhiyuan. Miao, Mingsan. \& Gu, Liya. 2008. Affect system Polygonum polysaccharide on immune function in mice. TCM Res.21(6):18.

[5] Wang, Linan. Wang, Qin. \& Miao, Mingsan. 2010. FCPS suppress immune function in mice with cyclophosphamide -induced immune. China journal of Chinese medicine. 4(25):677.

[6] Bai, Chengxu. Xu, Xiuju. \& Chang, Jiang. 2007. The effect of cactus on immune function of rats. Modern preventive medicine. 34(16):3064. 